\section{Brain, Behavior and Evolution}

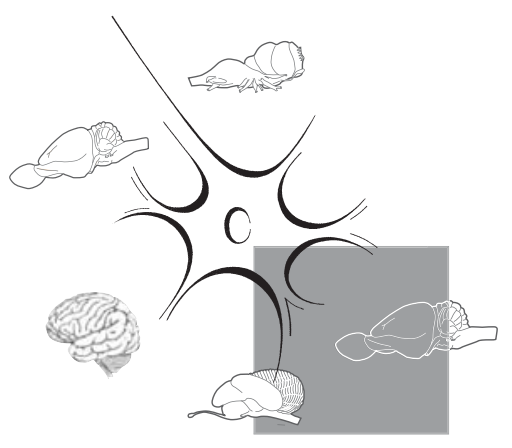

Weisbecker [2009] makes aggressive claims about our work linking evolution and development of the mammalian brain, without reviewing our corpus of work as it has evolved over the past 15 years. She argues that: (1) our analysis of mammalian developmental event timing speciously overinflates conservation of the developmental plan, (2) the sequence of neurogenesis does not predict structure size, and even if it did, (3) it would be uninteresting. She suggests heterochronic change, not conservation, is most informative about brain evolution. Although Weisbecker makes multiple, answerable claims about statistical analysis, due to space limitations, here we respond principally to evolutionary issues of most relevance to readers of Brain, Behavior and Evolution.

Since the time we published 'Linked regularities in the development and evolution of mammalian brains' [Finlay and Darlington, 1995], developmental evolutionary biology has undergone radical change. The stunning demonstrations of conserved organization of invertebratevertebrate body plans were just appearing. The significance of conserved systems for evolution began to be analyzed, shaping the new field of 'evo-devo'. In a pre-evodevo context, when we first described the strong relationship between brain allometry and neurogenesis - which seemed to make certain structures disproportionate-

Highlights and Perspectives on Evolutionary Neuroscience

Published online: March 8, 2010 DOI: $10.1159 / 000295350$

\title{
Late Still Equals Large
}

\author{
A Response to Weisbecker V (2009): Why 'Late Equals Large' Does Not \\ Work. Neuroscience 164:1648-1652
}

\author{
Barbara L. Finlay, Barbara Clancy, Richard B. Darlington
}

Department of Psychology, Cornell University, Ithaca, N.Y., USA

ly large by rule, independent of specific niches and behaviors - we struggled to account for it in terms of 'developmental constraints' and 'spandrels'. Now, we attempt to understand the conserved pattern of allometric scaling as a substrate for 'evolvability'. That is, we investigate what features such a conserved plan might afford for graceful scaling and facilitated variability (that is, genetic variation translated through conserved genetic and epigenetic contexts which coordinate and stabilize functionality), working this out in some detail for the nocturnal and diurnal primate eye. Of course, understanding behavioral variability remains the goal, but because relative volumes of specific neural structures rarely correlate well with specific capacities, most researchers have sensibly turned their attention elsewhere.

\section{Inflating Developmental Conservation}

Weisbecker treats 15 years of evolving analyses as a single entity. The original dataset included only neurogenesis events and placental mammals, with one possum [Finlay and Darlington, 1995]. Next we related neurogenesis events to brain segmentation [Finlay et al., 1998], and then we added several more metatherian animals, and non-neurogenesis events [Darlington et al., 1999]. Later still we added data per- mitting primate cortical and limbic correction factors [Clancy et al., 2001]. Our 'Translating Time' database (www.translatingtime.net) is continually remodeled, now with over 500 'event' datapoints. We invite readers to consult the cited papers to examine the variety of models evaluated and contrasted.

Weisbecker makes several arguments that the statistical methods we use overestimate variance explained and mischaracterize the variability of the data. Here we address 3 of the most central criticisms: that the model is 'circular' (tested on the same data from which it was derived), that modeling data as log transformation of days is misleading, and that the model is overdosed with systems whose orders are developmentally contingent on each other.

Circularity. We refuted this with the 'leave one out' technique [Finlay et al., 2001, p 302].

Log Transforms. In humans, is 'age first permanent teeth appear' more variable than 'age at birth' measured from conception? Most life history researchers agree that this question is best framed with a logarithmic scale of age, which automatically expresses variability as a proportion of the values being discussed. Oddly, Weisbecker proposes we compute the standard deviations of these 2 ages and then derive an 'average' standard deviation. 'Heteroscedasticity' means that the

\section{KARGER}

(C) 2010 S. Karger AG, Basel

Fax +41613061234 E-Mail karger@karger.ch www.karger.com www.karger.com/bbe
Barbara L. Finlay

Department of Psychology, Cornell University

Uris Hall

Ithaca, NY 14853 (USA)

Tel. +1 607255 6394, Fax +1 607255 8433, E-Mail blf2@ cornell.edu 
typical errors of prediction are larger for some values of the independent variables than for others, and if it is an unavoidable feature of one's data, then the appropriate procedure is to use different standard errors for different parts of the model or eliminate them by explicit transformations. Weisbecker does just the opposite, taking the large variability of the latestoccurring events in the slowest-developing species (humans), and applying these high variability figures to earlier events and to faster-developing species. Finally, if a single transformation can transform heteroscedasticity into homoscedasticity, and also transform nonlinearity into linearity, that's a double bonus. Our Y-transformation, the most biologically and statistically appropriate one for these data, did both, allowing computation of a single standard error of predictions that applies across the entire model.

Constraining Effects of 'Contingency'. Weisbecker supposes that the order of developmental trophic interactions, which she terms contingency, must be the same as the flow of information through the adult visual system (i.e. retina, thalamus, cortex) somehow overconstraining the data. Her misunderstanding points out the interesting feature that developmental sequences may be conserved even when the immediate consequences seem disadvantageous. In fact, cones, retinal ganglion cells, the thalamus and the initial cortical layers are generated first, immediately followed by retinal interneurons and cortical thalamorecipient zones, and finally, the upper layers of cortex and rod photoreceptors. The comparative consequences of this sequence actually caused consternation when neuroscientists noticed that thalamic neurons and their axons are generated early enough to reach the cortex well before their target cells arrive, requiring a 'waiting period' in the large brains of cats and monkeys for thalamic axons when they reach the cortical plate [Allendoerfer and Shatz, 1994]. In any case, as the database expands, continually changing the contribution of the systems represented in the first dataset, predictability has not dropped.

\section{Position in Neurogenesis and Structure Scaling}

One persistent problem comparing the allometric and developmental data sets is that different 'brain' units are used, typi- cally entire structure volumes in allometry studies, versus smaller nuclei, layers or identified cells in neurogenesis studies. Originally, we tested that the modeled 'structure score' of ordinal position in neurogenesis predicted the allometric scaling of the 7 brain regions which happened to be identical in both sets (of 51), and found a correlation of 0.943 [Finlay and Darlington, 1995]. Although Weisbecker does not seem to notice this analysis, to base such a large claim on this subset required more verification.

For that reason, we immediately investigated multiple predictions of this hypothesis in homochronic and heterochronic contexts. In 1995, we showed that monkey limbic system and cortex are respectively smaller and larger than allometrically predicted, with neurogenesis schedules correspondingly retarded and advanced. Then, we described a spatial correlate of duration of neurogenesis in the newly described prosomere model of the embryonic brain [Rubenstein et al., 1994] corresponding to structure scaling in the brain overall [Finlay et al., 1998]. Looking at a smaller scale, we computed weighted averages of birthdates of specific thalamic nuclei from Bayer and Altman's [1995] comprehensive rat neurogenesis data to predict the allometric scaling of homologous nuclei in anthropoid apes, again showing a good fit. We also had similar findings for amygdaloid nuclei in rats and their relative size in rhesus macaque [Finlay et al., 2001]. Recently, we described new data showing that extension of neurogenesis in the nocturnal owl monkey produced more numerous rods [Dyer et al., 2009]. We particularly draw attention to this work, which shows that the conserved order of retinal neurogenesis, in which the retinal cell classes relevant to nocturnal and diurnal vision are each grouped together, appears to allow a 1-parameter, coordinated shift of multiple retinal components from diurnal to nocturnal organization and number, as well as graceful scaling of cell classes for the optical requirements of eyes of different sizes [Finlay, 2008].

In Weisbecker's table 1 , she takes the data which we explicitly rejected for this use and averages together the ordinal scores of whatever few cell groups in the embryonic data set are located within larger allometric brain divisions, without correcting for the percentage of the structure's volume the groups comprise (e.g. she produces a score for the 'cerebellum' by av- eraging the ranks for Purkinje cells and deep cerebellar nuclei, omitting the granule cells of cerebellar cortex, its largest and latest-generated component). She then predicts the scaling values from these averages, a nonsense analysis.

\section{Preaching to the Preachers}

Weisbecker's most disturbing allegation is that simply because we have observed a high predictability in allometric scaling and developmental sequencing, we are committed to believe conservation is the only important aspect of brain evolution. In fact, we believe it is a powerful way to contextualize other developmental changes, allowing better focus on mechanisms producing diversity, including, but not limited to heterochrony. Studies of the roles of neuron loss and axon retraction in brain evolution originally suggested these neurogenesis studies. We have examined heterochrony in the macaque cortex/limbic system [Finlay and Darlington, 1995] and the owl monkey retina [Finlay, 2008; Dyer et al., 2009]. We are presently examining segmental boundary positions between the neocortex and limbic system in primate versus rodent. We hypothesized cell respecification and a new migratory path occurring within conserved neuron numbers in the primate pulvinar [Chalfin et al., 2007], and pointed out another case where primate cortical GABAergic cell production has an unpredicted time course [Clancy et al., 2009]. We suggest dynamic re-allocation of cortical volume rather than cortical area respecification in nocturnal and diurnal mammals [Kaskan et al., 2005]. We point to altered receptor expression in subcortical motivational systems as a way of configuring the contents of massive short- and long-term memory systems of cortex and hippocampus [Syal and Finlay, in press]. Beginning from 'late equals large', other researchers show that the brains of altricial birds (e.g. song birds) follow the general mammalian pattern, while precocial birds, behaviorally competent on hatching, segment and accelerate their brain neuron production differently [Charvet and Striedter, 2009].

Finally, we do insist that the function served by conserved, grossly disproportionate but predictable brain scaling is an important question, not the disparaged 'null hypothesis': it is not an obvious 'de- 
fault' to allow late-generated components of the cerebellum and telencephalon to increase so strikingly with respect to the rest of the brain. Understandably, the first evolutionary neurobiologists worked with the fairly crude theory of the brain that particular functions resided in particular structures, and that evolution could improve functions by selecting on the relative sizes of their committed structures. Our growing understanding of evolvable computational architectures, that is, neural architectures which allow additions and deletions of computational power and new functionality while leaving core functions undisturbed, is replacing this first theory. We speculate that the central problem in brain evolution that 'late equals large' works to solve is the maintenance of an evolvable neural architecture.

\section{Acknowledgements}

Recent work cited in this article was supported by the National Science Foundation grant IBN-0138113 to B.L.F., by NIH grant number P20 RR-16460 from the IDeA Networks of Biomedical Research Excellence (INBRE) Program of the National Center for Research Resources to B.C., NSF DBI 0849627 to B.C., and NSF DBI 0848612 to B.L.F.

\section{References}

Allendoerfer KL, Shatz CJ (1994): The subplate, a transient neocortical structure. Annu Rev Neurosci 17:185-218.

Bayer SA, Altman J (1995): Neurogenesis and neuronal migration: principles of neurogenesis, neuronal migration and neural circuit formation; in Paxinos G (ed): The Rat Nervous System, ed 2. Sydney, Academic Press, pp 1041-1098.

-Chalfin BP, Cheung DT, Muniz JAPC, Silveira LCL, Finlay BL (2007): Scaling of neuron number and volume of the pulvinar complex in New World primates: comparisons with humans, other primates and mammals. J Comp Neurol 504:265-274.
Charvet CJ, Striedter GF (2009): Developmental basis for telencephalon expansion in waterfowl: enlargement prior to neurogenesis. Proc Roy Soc B 276:3421-3427.

Clancy B, Darlington RB, Finlay BL (2001): Translating developmental time across mammalian species. Neurosci 105:7-17.

Clancy B, Teague-Ross T, Nagarajan R (2009): Cross-species analyses of the cortical GABAergic and subplate neural populations. Front Neuroanat 3:20.

Finlay BL (2008): The developing and evolving retina: using time to organize form. Brain Res 1192:5-16.

- Finlay BL, Darlington RB (1995): Linked regularities in the development and evolution of mammalian brains. Science 268:15781584 .
Finlay BL, Darlington RB, Nicastro N (2001): Developmental structure in brain evolution. Behav Brain Sci 24:263-308.

Finlay BL, Hersman MN, Darlington RB (1998): Patterns of vertebrate neurogenesis and the paths of vertebrate evolution. Brain Behav Evol 52:232-242.

Kaskan PM, Franco EC, Yamada ES, Silveira LCL, Darlington RB, Finlay BL (2005): Peripheral variability and central constancy in mammalian visual system evolution. Proc Biol Sci 272:91-100.

Rubenstein JLR, Martinez S, Shimamura K, Puelles L (1994): The embryonic vertebrate forebrain: the prosomeric model. Science 266:578-580.

Weisbecker V (2009): Why 'late equals large' does not work. Neuroscience 64:1648-1652. 